\title{
Attosecond Pump-probe Spectroscopy of Charge Dynamics in Tryptophan
}

Manuel Lara-Astiaso', Mara Galli ${ }^{23,}$, Andrea Trabattoni', Alicia Palacios', David Ayuso', Fabio

Frassettos, Luca Poletto ${ }^{s}$ Simone De Camillis ${ }^{\varsigma}$, Jason Greenwood ${ }^{\varsigma}$, Piero Decleva ${ }^{7}$, Ivano

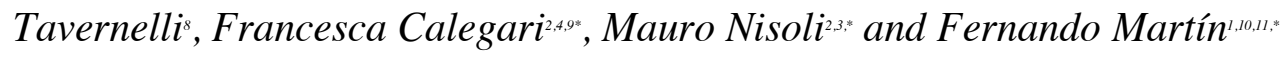

' Departamento de Química, Módulo 13, Universidad Autónoma de Madrid, 28049 Madrid, Spain

${ }_{2}^{2}$ Institute for Photonics and Nanotechnologies, IFN-CNR, Milano, Italy

${ }^{3}$ Dipartimento di Fisica, Politecnico di Milano, Milano, Italy

${ }^{4}$ Center for Free-Electron Laser Science (CFEL), DESY, Hamburg, Germany

${ }^{5}$ Institute for Photonics and Nanotechnologies, IFN-CNR, Padova, Italy

'School of Maths and Physics, Queen's University, Belfast, UK

' Dipartimento di Scienze Chimiche e Farmaceutiche, Universitá di Trieste, 34127 Trieste, Italy

${ }^{8}$ IBM Research GmbH, Zurich Research Laboratory, 8803 Rueschlikon, Switzerland

${ }^{9}$ Department of Physics, Hamburg Universität, Hamburg, Germany

${ }^{10}$ Instituto Madrileño de Estudios Avanzados en Nanociencia, 28049 Madrid, Spain 
"Condensed Matter Physics Center (IFIMAC), Universidad Autónoma de Madrid, 28049 Madrid, Spain

Equally contributed

*Corresponding authors: $\quad$ francesca.calegari@desy.de, mauro.nisoli@polimi.it and fernando.martin@uam.es

ABSTRACT:

Attosecond pump-probe experiments performed in small molecules have allowed tracking charge dynamics in the natural time scale of electron motion. That this is also possible in biologically relevant molecules is still a matter of debate, since the large number of available nuclear degrees of freedom might destroy the coherent charge dynamics induced by the attosecond pulse. Here we investigate XUV-induced charge dynamics in the aminoacid tryptophan. We find that, although nuclear motion and non-adiabatic effects introduce some decoherence in the moving electron wave packet, these do not significantly modify the coherence induced by the attosecond pulse during the early stages of the dynamics, at least for molecules in their equilibrium geometry. Our conclusions are based on elaborate theoretical calculations and the experimental observation of sub-4 fs dynamics, which can only be reasonably assigned to electronic motion. Hence, attosecond pumpprobe spectroscopy appears as a promising approach to induce and image charge dynamics in complex molecules. 
TOC GRAPHICS

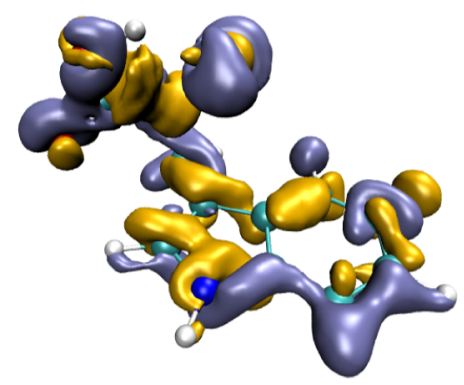


Imaging electron dynamics in molecules with few-fs or sub-fs time resolution is the very first step towards achieving control of chemical processes that occur on a longer time scale [1-9]. The rapid progress in attosecond technology [10-14] has already permitted electron dynamics in atoms $[15,16]$ and simple molecules $[17,18]$ to be imaged and controlled, and similar behavior in more complex molecules such as biologically relevant molecules can be expected $[5,8]$.

A convenient approach to investigate electron dynamics is attosecond pump-probe spectroscopy $[1,5-7,17-20]$. Among the different strategies proposed in the literature, of special interest is the case in which an attosecond extreme ultraviolet (XUV) pump pulse, e.g., produced by highharmonic generation, ionizes the molecule and a time-delayed few-fs near-infrared (NIR) pulse is used to probe the charge dynamics generated in the molecular cation by the pump pulse. The first and, to our knowledge, only experimental work that has provided real-time evidence of charge dynamics in a relatively large molecule using this technique was reported in 2014 for the amino acid phenylalanine [5]. In this work, the fragmentation yield associated with the production of the doubly-charged immonium ion exhibits few-fs oscillations superimposed to a smooth decreasing background typical of the much slower nuclear dissociation dynamics. With the help of theoretical calculations performed within the static exchange density functional theory (DFT) and the fixednuclei approximation, these fast oscillations were interpreted as the signature of the coherent electron dynamics generated by the attosecond pulse on the remaining molecular cation [5,21].

A rather unexpected result of this work was the realization that the observed electron dynamics can be explained, on the few-fs time scale, by just a few characteristic frequencies, despite the large number of molecular states excited by the broadband attosecond pump pulse. This remarkable observation strongly supports the use of attosecond techniques to initiate and image charge migration/transfer processes in large molecules. However, to fully get into this path, one 
has first to check if similar simple charge dynamics can be induced in other molecules, especially larger ones, and then elucidate to what extent the degree of coherence of the electronic dynamics initiated by prompt ionization is affected by the nuclear degrees of freedom. The experimental observation of few-fs features in phenylalanine was a first indication that, at least in particular cases, dephasing due to nuclear motion may not necessarily prevent the observation of ultrafast coherent electron dynamics. However, there is no guarantee that this would always be the case or that the coupling with the increasing number of nuclear degrees of freedom present in large molecules, giving rise to possible non-adiabatic relaxation pathways close to the Frank-Condon region, would not completely wash out the coherence of the electronic wave packet just after its creation [22-24].

With the aim of going deeper into these questions, we have performed elaborate theoretical calculations in combination with attosecond pump-probe experiments on the tryptophan molecule. Besides accurately describing the ionization step, which is necessary to obtain a realistic description of the propagating electronic wave packet, the present theoretical model takes into account the effects of both electron correlation and nuclear motion, which have been previously disregarded (Ref. [5]). This is very challenging for such a large molecule, since one must evaluate the ionization amplitudes (modulus and phase) for a large number of open channels.

Tryptophan (Trp) has been chosen among the twenty known amino acids, because it is the one with the most interesting photochemical properties. Indeed, its chromophore, the indole group (an aromatic heterocycle formed by a pentagonal and a hexagonal ring, see Fig. 1), is responsible for the largest part of the UV-VUV absorption of proteins, and its characteristic absorption and emission bands are very sensitive to its local environment. Thus, Trp is currently used to probe protein folding, to investigate the mechanism of electron transfer through the protein matrix and 
to monitor electron and proton dynamics in photo-excited states [25-27]. Therefore, time resolving electron dynamics in this molecule may be useful to extend the applicability of Trp-based photochemical techniques to the attosecond time domain.

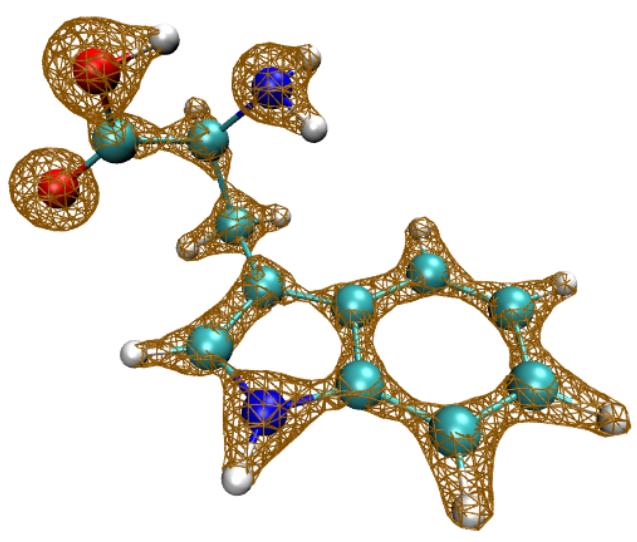

Figure 1. Structure of the most abundant conformer of tryptophan. Green spheres: carbon atoms; blue spheres: nitrogen atoms; red spheres: oxygen atoms; white spheres: hydrogen atoms. The hole electron density created by the attosecond pulse used in the experiment is represented by a brown mesh (isovalue $\left.=-0.003 \mathrm{bohr}^{3}\right)$.

In this paper, we show that irradiation of tryptophan by an XUV attosecond pulse generates fewfs electron dynamics that must arise from the coherent superposition of the valence states of the molecule. By just involving valence states in the ionization step, one avoids Auger decay processes, which is an important source of damage in large molecules, thus favoring the persistence of the induced charge dynamics in the original (unbroken) molecule. The generated electron dynamics leads to significant charge fluctuations in the indole group, which could thus be used to probe similar electron dynamics occurring in the Trp environment. Finally, and most importantly, we show that, for molecules in their equilibrium geometry, nuclear motion and non- 
adiabatic effects do introduce some decoherence in the propagating electronic wave packet, but this is not enough to significantly modify the coherent charge dynamics ignited by the attosecond pulse, at least during the first $20 \mathrm{fs,}$, when several cycles of charge fluctuations have already occurred. Hence, attosecond pump-probe spectroscopy appears as a general and promising approach to induce and image charge dynamics in complex molecules.

Let us start by describing the actual electronic wave packet that is formed by irradiating Trp with the XUV attosecond pulse used in the experiment, whose spectrum is shown in Fig. 2(a). We have theoretically evaluated such a wave packet and its ulterior time evolution for the most abundant conformer of Trp in its equilibrium geometry by using a methodology that goes well beyond that employed in Ref. [5]. In particular, the ionization amplitudes that define the coherent superposition just after the ionization step have been obtained by using the full-electron static-exchange density functional theory (DFT) method [28,29], which makes use of localized B-spline functions. This method has been previously applied to describe ionization of complex molecules, among them glycine and phenylalanine [5,30-32], with great success. The photoionization cross sections (proportional to the squared moduli of the ionization amplitudes) for the 39 ionization channels accessible with the attosecond pulse are shown in Fig. 2(a). As can be seen, in the range of photon energies covered by the attosecond pulse, all accessible channels have comparable cross sections (roughly within a factor of 3) and are thus expected to contribute to the coherent superposition. To gauge the quality of these calculations, in Fig. 2(b) we compare the theoretical total photoelectron spectrum with the only one available in the literature from synchrotron radiation measurements at a photon energy of $100 \mathrm{eV}$ [33]. The agreement is reasonably good. 

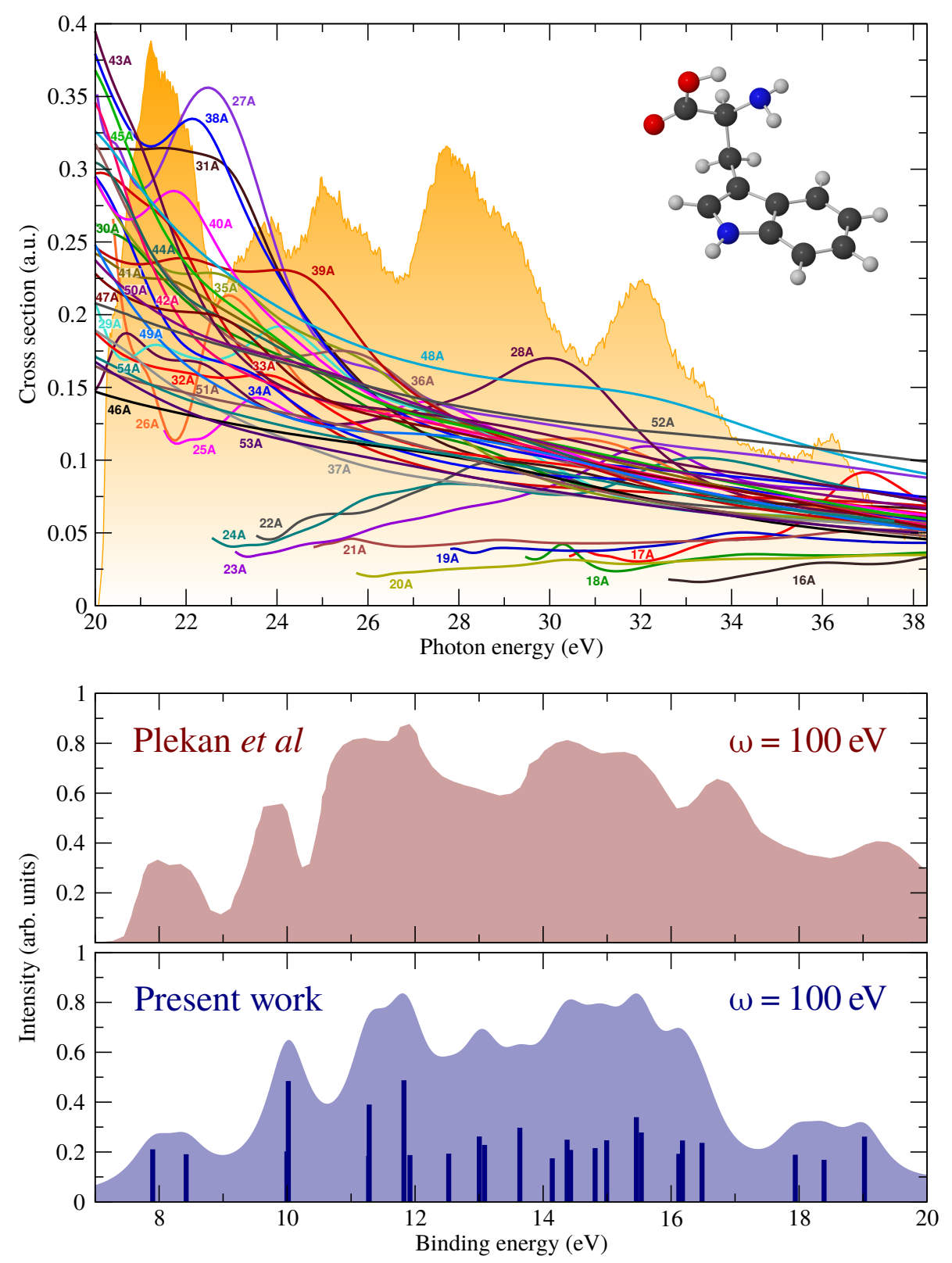

Figure 2. (a) Photoionization cross sections of Tryptophan. The labels indicate the molecular orbital from which the electron is removed (54A denotes the HOMO, 53A the HOMO-1, and so on). The yellow curve over the shaded area shows the spectrum of the attosecond pulse used in the experiment. (b) Comparison between the measured photoelectron spectrum [33] and the calculated one at $100 \mathrm{eV}$ (lower panel). For a realistic comparison, our infinitely resolved spectral lines have been convoluted with a Lorentzian function of $0.4 \mathrm{eV}$ full width at half maximum (FWHM) that reproduces the experimental broadening of the peaks. 
Figure 1 shows the hole density (equivalent to the charge density) at time zero, i.e., just after interaction with the attosecond pulse. This hole density results from the coherent superposition of a large number of electronic one-hole states and is therefore very delocalized. The subsequent field-free evolution of the hole wave packet has been described by using an extension of timedependent DFT in combination with Ehrenfest molecular dynamics (TDDFT-ED) as implemented in the CPMD package [34] (see Methods). At variance with earlier work on phenylalanine [5], in our TDDFT-ED approach, the evolution of the electronic density is no longer described as a superposition of single-hole configurations, as the time dependence of the density is explicitly built into the propagation of the TDDFT equations. Thus, variations due to changes in electron correlation during the time evolution are allowed. Moreover, decoherence effects due to nuclear motion and non-adiabatic effects are taken into account via Ehrenfest dynamics. The simulations have been performed for a molecule that is initially in its equilibrium geometry (i.e., single trajectory). Figure 3 shows snapshots of the calculated hole density at different times, for both the case in which the positions of the nuclei are fixed and the case in which they are allowed to move. To better visualize temporal changes in the density, the latter has been referred to its mean value over the time interval chosen to perform the calculations. As can be seen, very fast charge fluctuations occur around any molecular site, with no preference for specific functional groups. Thus, contrary to chemical intuition, for a few hundreds of attoseconds, functional groups with large electron affinity may bear less electronic charge than functional groups with less or no electron affinity, thus transiently leading to profound changes in the chemical bonding. In particular, charge fluctuations are rather apparent around the indole chromophore, where regions exhibiting an excess of charge (purple) rapidly turn into regions with a deficit of charge (yellow). This result suggests that attosecond resolved photoemission and/or photoabsorption experiments 
performed at wavelengths where the indole chromophore is active (e.g., by using attosecond transient absorption spectroscopy techniques [35]) might be useful to provide temporal information about charge dynamics occurring in the Trp environment, e.g., in a large protein.

\section{Fixed nuclei}
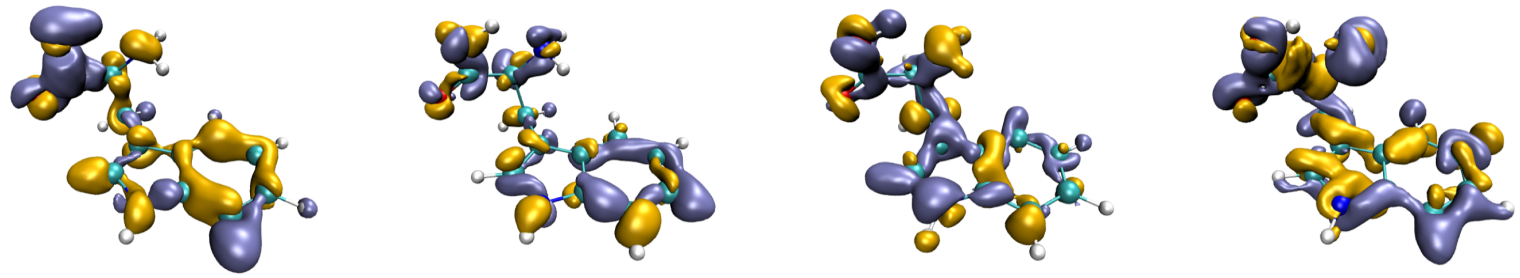

Moving nuclei

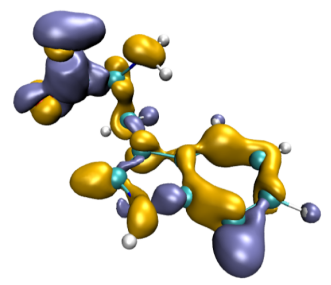

$0 \mathrm{fs}$

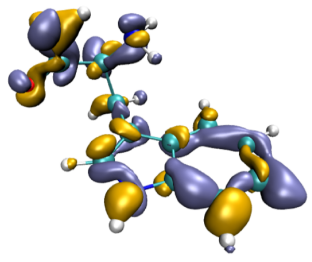

$3 \mathrm{fs}$

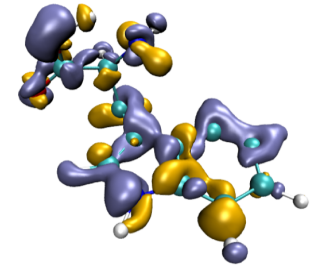

$6 \mathrm{fs}$

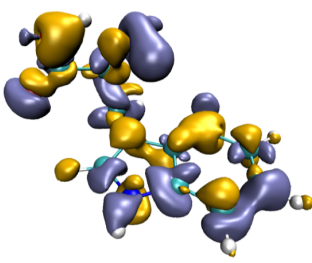

9 fs

Figure 3. Snapshots of the charge density evolution. Upper row: results obtained by fixing the positions of the nuclei. Lower row: results obtained by allowing the nuclei to move as described by the Ehrenfest MD formalism. For a better visualization, at all times the hole density is referred to its average value over the complete time interval. Regions with an excess or a deficit of charge with respect to this average value are represented in purple and yellow colors, respectively.

To experimentally access the dynamics of this electronic wave packet, gas-phase Trp molecules (with an internal temperature of approximately $450 \mathrm{~K}$ ) were irradiated by an isolated XUV attosecond pulse identical to that used in the above theoretical calculations. Then the ensuing charge dynamics was probed by a waveform-controlled $800-\mathrm{nm}$ sub- 4 fs pulse. A clean plume of 
isolated and neutral molecules was generated by evaporation of Trp from a thin metallic foil heated by a continuous wave $(\mathrm{CW})$ laser. The parent and fragment ions resulting from the interaction with the pump and probe pulses were analyzed by using a linear time-of-flight mass spectrometer (see [5] for details). Photoionization of a large molecule such as Trp by XUV pulses inevitably leads to the production of several cationic fragments. Previous work on phenylalanine $[5,19]$ has shown that ultrafast oscillations observed in the yield corresponding to the breakup into a carboxyl radical and a doubly charged immonium ion are most likely associated with charge fluctuations around the amino group, which remains bonded to the central body of the molecule and therefore can keep exchanging charge with the latter while the carboxyl group is leaving or has completely left. Here we follow a similar strategy and concentrate on the equivalent doubly charged ionic fragment [Trp+ "minus" the $\mathrm{COOH}$ radical, or (Trp-COOH)+ for short], where, according to Fig. 3, charge exchange between the amino group and the rest of the molecule should follow a similar pattern.

Fig. 4 shows the yield for the production of (Trp-COOH)+(mass/charge ratio of 79.5) as a function of the pump-probe delay. The most prominent feature of the measured yield is the sharp increase from zero time delay up to about $12 \mathrm{fs}$, followed by a steady decay that extends beyond $30 \mathrm{fs}$. As discussed in [19], this reflects the nuclear dynamics induced by the XUV pulse, but also by the NIR probe pulse, which further ionizes the molecule and leads to its dissociation in the fs time scale. Superimposed to this variation of the yield, one can clearly distinguish oscillations that occur on a much shorter time scale. By appropriately subtracting the slow varying background, the oscillations are more clearly revealed and a fit to sinusoidal functions could be easily performed (see inset in Fig. 4 and details in the Supporting Information). From this fit, we have obtained the main characteristic frequency of this ultrafast dynamics: $0.252 \pm 0.011 \mathrm{PHz}$, corresponding to a 
period of $4 \mathrm{fs}$. The presence of a single frequency already suggests that, as in the phenylalanine case [5], the ultrafast dynamics induced in Trp by the attosecond pulse is relatively simple.

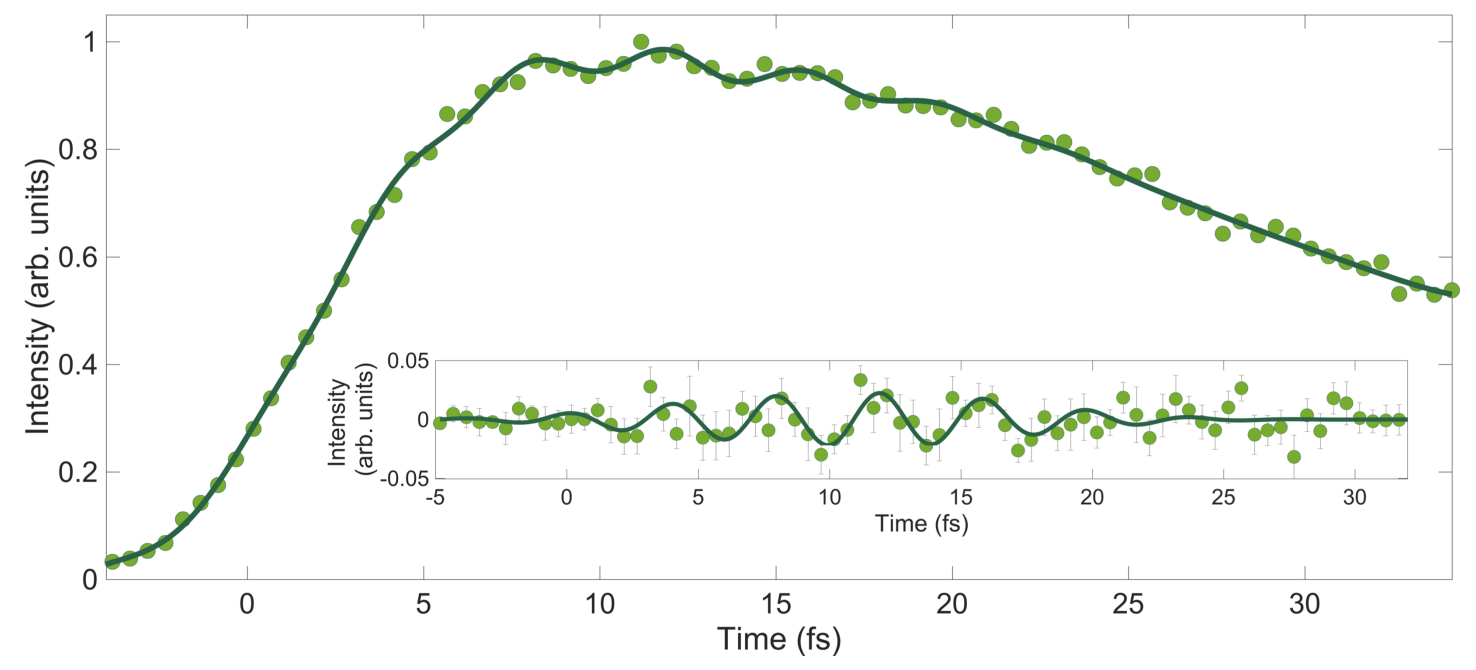

Figure 4. Integrated yield of the doubly charged immonium ion (mass/charge $=79.5$ ) as a function of XUV pump - NIR probe delay (dotted line). The black line is a fitting curve used to guide the eye. Inset: differential signal obtained by subtracting a fitting function from the data. The black curve is a sinusoidal function of frequency $0.252 \mathrm{PHz}$. Error bars show the standard error of the results of two measurements.

We have extracted additional dynamical information by performing a time-window Fourier transform of both the measured yield and the calculated hole-density around the amino group. The results are shown in Fig. 5. For comparison, we also show the results obtained in the fixed-nuclei approximation. As can be seen, the effect of nuclear motion, though not negligible, does not lead to dramatic changes in the calculated frequencies. In other words, for the molecule under investigation, nuclear motion and non-adiabatic effects do not seem to substantially modify the initial coherence of the wave packet or alter the simple picture that only a few frequencies are responsible for the observed dynamics. For a meaningful comparison with the experimental results 
shown in Fig. 5(d), the calculated spectrum has been convoluted with a gaussian filter function that accounts for the time resolution of the experiment, leading to the results shown in Fig. 5(c). The same filter has been used to plot the experimental results. The frequency observed experimentally at $0.252 \mathrm{PHz}$ corresponds to the slower beating retrieved from the theoretical calculations and it exhibits a similar time behavior, gradually disappearing after 25 fs from zero time delay. The faster beating predicted by theory at $0.53 \mathrm{PHz}$ (see Fig. 5(a)) is not seen in the experiment as a consequence of the limited time resolution. This important result confirms that, even for a very large molecule such as Trp, electronic coherences may survive nuclear motion.

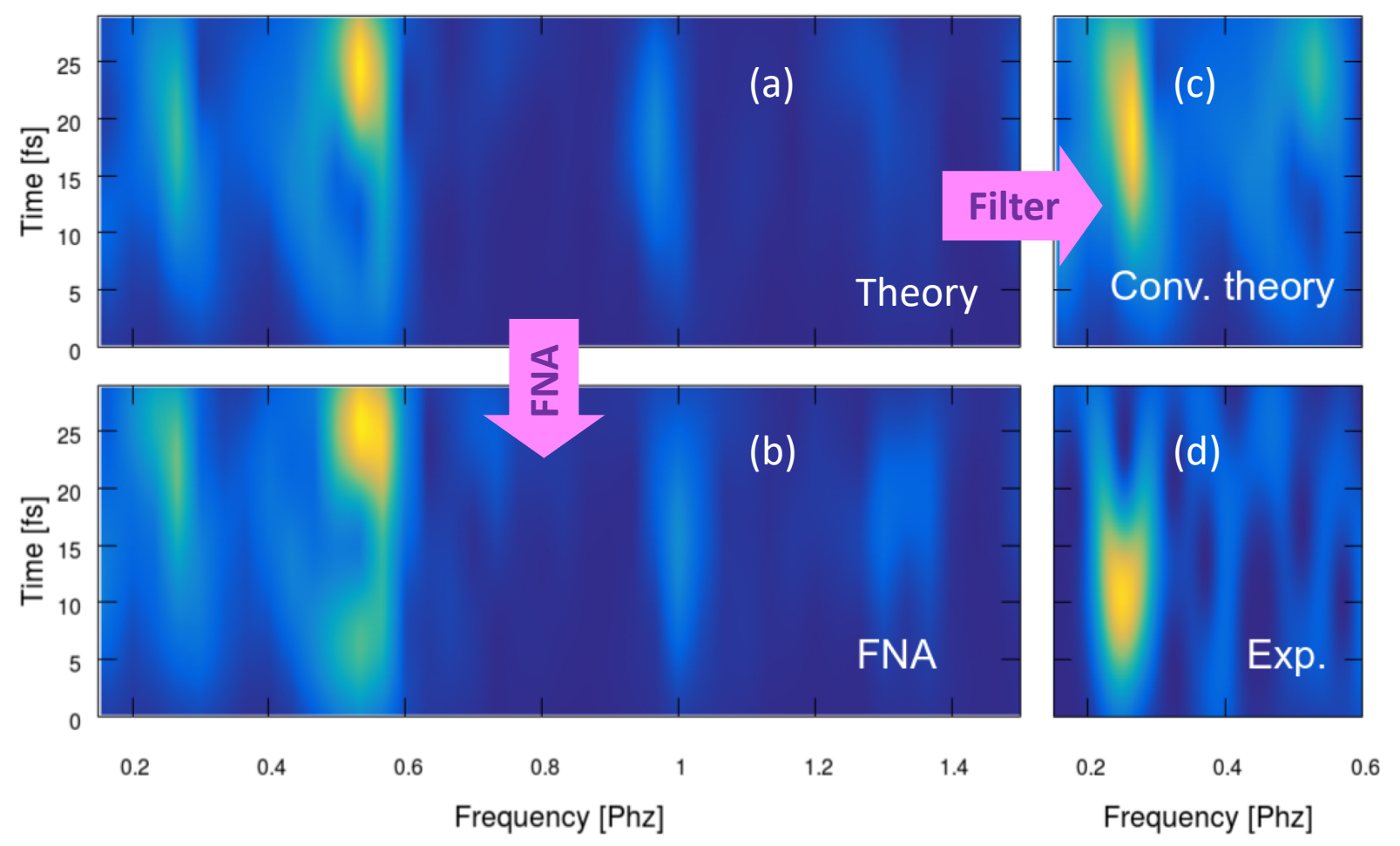

Figure 5. (a) Time-window Fourier transform of the electronic density generated by the attosecond pulse around the amino group in Trp. (b) The same within the fixed-nuclei approximation, FNA. (c) Convolution of the results given in (a) with a gaussian filter function with a fwhm of $7 \mathrm{PHz}$ that accounts for the time resolution of the experiment. (d) Time-window Fourier transform of the measured fragmentation yield shown in Fig. 4. 
A careful inspection of Fig. 5 shows that the main feature at $0.25 \mathrm{PHz}$ appears earlier in the experiments than in the calculations. This might be due to modifications of the original dynamics by the NIR probe pulse and/or the ensuing fragmentation dynamics, which are not described in the theoretical simulations. Also, one cannot completely discard a systematic error of about 1-2 fs in the calibration of the experimental pump-probe delay.

To get additional insight on the role of non-adiabatic effects, we have projected at different times the time-dependent Kohn-Sham (KS) orbitals onto the stationary KS orbitals. The results are shown in Fig. 6 for a few characteristics orbitals. In most cases, the calculated projections simply oscillate around their initial values, thus indicating that the population of the corresponding molecular orbitals remains more or less the same for all times and, therefore, do not significantly alter the evolution of the electronic wave packet. This is the case for the $\Phi_{16}$ orbital shown in Fig. 6. In contrast, for some other orbitals, like the $\Phi_{18}$ one, non-adiabatic transitions associated with a change in the orbital character can be observed. These occur after approximately $15-20$ fs and, therefore, cannot play any role during the early stages of the wave packet evolution. Nevertheless, as non-adiabatic transitions show up at times in which oscillations in the measured yields are still visible, they do have some effect in the relative intensities and positions of the recorded frequencies (compare Figs. 5(a) and 5(b)). These findings are in agreement with those recently reported for glycine by using a similar theoretical treatment [36]. The calculated projections also show that the KS orbitals involved in the beating appearing at around $0.25 \mathrm{PHz}$ are delocalized all over the molecule, so that they play an active role in charge fluctuations around the indole group (see Fig. $3)$. 
Due to the complexity of the theoretical treatment used in the present work, our analysis of the effect of the coupled electron and nuclear dynamics on the induced coherences has been limited to a Trp molecule in its equilibrium geometry. However, recent theoretical work [23] has reported that the evolution of the electronic wave packet corresponding to different initial nuclear positions, compatible with the molecular geometries expected in the Franck-Condon region, could be slightly different, thus leading to a damping of the charge fluctuations, and possibly to a broadening of the associated frequencies. The present experimental results reveal that, at least in the present case, these effects might not be so pronounced as to completely wash out the electronic coherences.
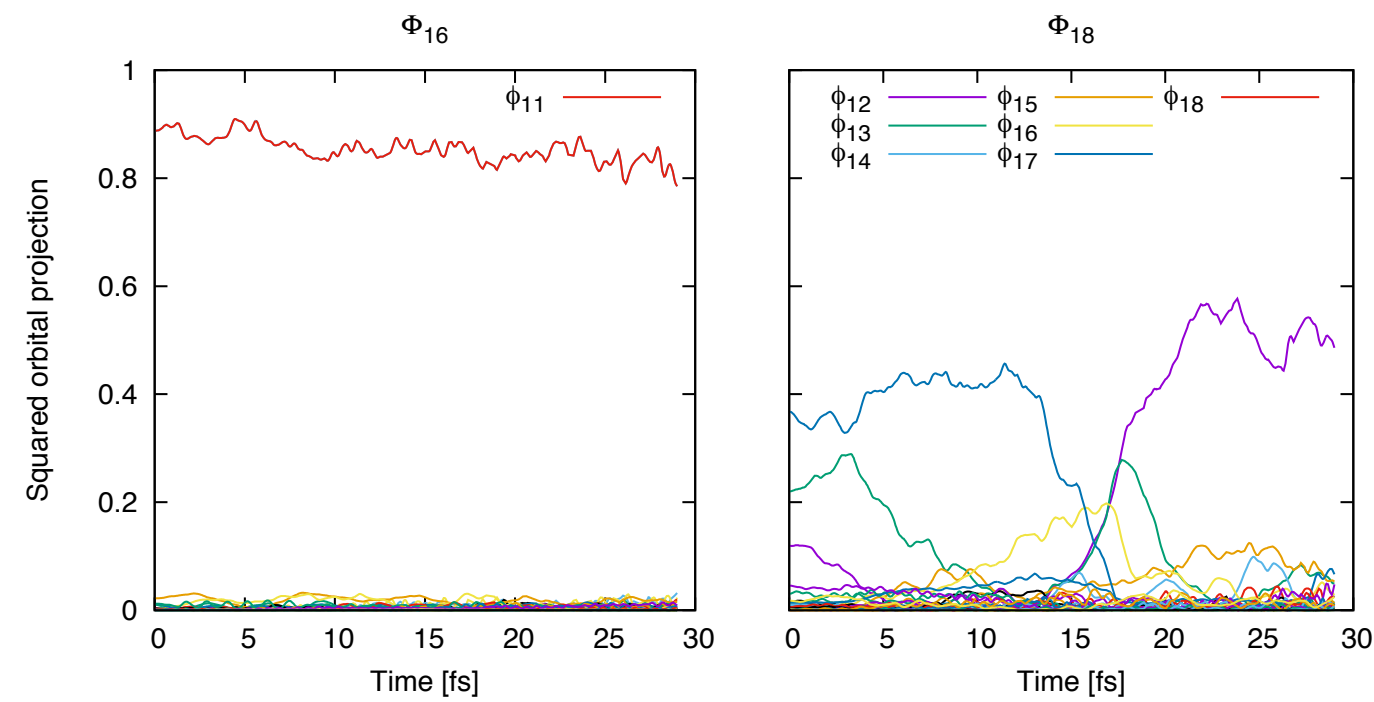

Figure 6. Projections of selected time-dependent KS orbitals $\left(\Phi_{16}\right.$ and $\left.\Phi_{18}\right)$ onto the stationary KS orbitals $\phi$ as a function of time.

In conclusion, we have performed a combined theoretical and experimental investigation in tryptophan that suggests the existence coherent electron dynamics associated to a few characteristic frequencies, thus confirming that this may be a rather general scenario in complex molecules of similar size, in particular biologically relevant molecules. The key result here is that 
electronic coherences, responsible for the ultrafast oscillations observed in fragmentation yields, are not fully destroyed by nuclear motion and non-adiabatic transitions during the wave packet evolution, at least for molecules in their equilibrium geometry. Both effects are common and relevant in large molecules due to the large number of available electronic and nuclear degrees of freedom, and the presence of hydrogen atoms, which move fast. The results of the present work suggest that they take place after electronic coherences have been established, so that the latter have enough time to leave their signature in the measured fragmentation yields.

While these findings indicate the suitability of ultrashort pulses to capture ultrafast dynamics and, in the long-term, pave the way to achieve certain control on the subsequent chemical path, a few more questions remain open to discussion, as e.g., why the probe pulse does not seem to significantly modify the frequencies associated with the electronic wave packet. Recent simple theoretical models have suggested that an XUV probe pulse could introduce new characteristic frequencies, mostly out of the range that is experimentally accessible, while preserving the original frequencies [36]. However, there is no theoretical evidence yet that this should also be the case for a NIR probe pulse. Including the effect of the NIR probe pulse in molecular systems of this size is extremely demanding, as one should evaluate multi-photon (not just one-photon) ionization of the singly-charged molecular cation from all states entering the coherent superposition created by the attosecond pulse (39 states in Trp) to all energetically accessible final states leading to a molecular di-cation (typically, many hundreds). And this for all the pump-probe delays, which imply different geometries.

Another open question is why the superposition of electron dynamics generated from different molecular geometries, as e.g. those contained in the Franck-Condon region, do not seem to blur the dynamics arising from a well-defined molecular geometry. Answering these questions in large 
molecules calls for even more refined theoretical methods and detailed experiments, which should be available in the near future.

Finally, it is important to stress that, although the use of XUV attosecond pulses inevitably leads to ionization and, therefore, the investigated charge dynamics is that of the remaining molecular cation, there are current experimental efforts to produce similar attosecond pulses in the UV, which would not ionize the molecule and thus would be ideal to investigate charge dynamics in neutral systems. In this case, the broadband UV pulse would also lead to a coherent superposition of electronic states, this time in the neutral molecule, which would necessarily lead to fluctuations in the electron density similar to those described in the present work. This electron dynamics could similarly be affected by nuclear dynamics and the passage through conical intersections as well. Therefore, all the knowledge gained by using XUV attosecond pulses could be directly extrapolated to few-fs or attosecond UV pulses.

\section{THEORETICAL METHODS}

We have carried out the calculations for the free propagation of the electronic density after interaction with the attosecond pulse by using the plane-wave Kohn-Sham (KS) based DFT methodology implemented in the CPMD package [34], in which nuclear dynamics is treated within the Ehrenfest formalism. It is well known that Ehrenfest dynamics cannot capture nuclear wave packet branching. However, in the present case, this effect plays essentially no role in the description of the dynamics during the first 10 fs following ionization, and only contributes moderately at later times. This is because, for this system and under the present conditions, the nuclear wave packet cannot diffuse into different regions of the nuclear configuration space in such a short time interval. Ehrenfest dynamics accounts for nuclear wave packet splitting in a mean 
field level, which is accurate enough when the different surfaces do not produce a significant spread of such nuclear wave packet in space.

Preparation of the initial electronic density has been performed exactly as described in previous work [36]. The TDDFT simulations were performed, within the frozen core approximation, by using a tetragonal box of dimensions 30x20x20 Å containing a single Trp molecule. Following the standard approach, core electrons were replaced by pseudo-potentials of the normal TroullierMartins form [38]. The use of pseudopotentials is justified because, even for the largest photon energies contained in the XUV pulse, core electrons are not accessible (for this, one would require photons of several hundreds of eV). Also, involvement of core electrons in the free propagation of the electron density is very unlikely, if not totally impossible, because correlation between core and valence electrons is negligible. The plane-wave basis set employed was truncated at an energy cutoff of 70 Ry. For both pseudo-potentials and active electrons, the energies and forces were evaluated using the Kleinman-Bylander scheme [39]. The exchange-correlation potential was represented by using the generalized gradient approximation PBE functional, which has been shown to accurately describe electronically excited states of Trp [40] and is computationally more convenient than the LB94 one used in the static exchange DFT calculations when a basis of plane waves is used. Preliminary studies performed on glycine using hybrid functionals did not show any significant improvement of the results compared to DFT/TDDFT/PBE. In this respect, it is important to emphasize that we are interested in studying the electronic relaxation of a perturbed electronic structure, which (in the time scale of our calculations) does not produce any bond breaking (that would require a good description of static correlation). Electronic relaxation in molecular systems is dominated by dynamical (and not static) correlation, which in general is well described by DFT/TDDFT/PBE calculations. 
We have projected the KS orbitals obtained from the static exchange DFT calculations (defining the electronic density) into the KS orbitals that initialize the time-dependent Kohn-Sham propagation in order to consistently define a new reduced density matrix. The numerical integration that solves the equations of motion with the CPMD code is performed using an iterative scheme based on a two-step Runge-Kutta propagator [41], which maintains order $\delta t^{3}$ accuracy. The used time step, $\delta \mathrm{t}=0.01$ atomic units $(0.241$ as), ensures energy conservation over the entire simulation range ( $25 \mathrm{fs}$ ) with a maximum deviation at the end of the simulation is $0.2 \mathrm{eV}$.

\section{AUTHOR INFORMATION}

The authors declare no competing financial interests.

\section{ACKNOWLEDGMENT}

Work supported by the MINECO project FIS2016-77889-R. We acknowledge computer time from CCC-UAM and Marenostrum Supercomputer Center. FM acknowledges support from the 'Severo Ochoa' Programme for Centres of Excellence in R\&D (MINECO, Grant SEV-2016-0686) and the 'María de Maeztu' Programme for Units of Excellence in R\&D (MDM-2014-0377).

SUPPORTING INFORMATION AVAILABLE: Fit of the experimental data.

\section{REFERENCES}

[1] Nisoli, M.; Decleva, P.; Calegari, F.; Palacios, A.; and Martín, F. Attosecond electron dynamics in molecules, Chem. Rev. 2017, 117, 10760-10825. 
[2] Cederbaum, L. and Zobeley, J. Ultrafast charge migration by electron correlation, Chem. Phys. Lett. 1999, 307, 205-210.

[3] Remacle, F. and Levine R. D. An electronic time scale in chemistry, Proc. Natl. Acad. Sci. USA 2006, 103, 6793-6798.

[4] Hennig, H.; Breidbach, J.; and Cederbaum, L. S. Electron correlation as the driving force for charge transfer: charge migration following ionization in N-methyl acetamide, J. Phys. Chem. A 2005, 109, 409-414.

[5] Calegari, F.; Ayuso, D.; Trabattoni, A.; Belshaw, L.; De Camillis, S.; Anumula, S.; Frassetto, F.; Poletto, L.; Palacios, A.; Decleva, P.; Greenwood, J. B.; Martín, F.; and Nisoli, M. Ultrafast electron dynamics in phenylalanine initiated by attosecond pulses, Science 2014, 346, 336-339.

[6] Lépine, F.; Ivanov, M. Y.; and Vrakking, M. J. J. Attosecond molecular dynamics: fact or fiction?, Nature Photonics 2014, 8, 195-204.

[7] Leone, S. R.; McCurdy, C. W.; Burgdörfer, J.; Cederbaum, L. S.; Chang, Z.; Dudovich, N.; Feist, J.; Greene, C. H.; Ivanov, M.; Kienberger, R.; Keller, U.; Kling, M. F. ; Loh, Z.-h.; Pfeifer, T.; Pfeiffer, A. N. ; Santra, R.; Schafer, K.; Stolow, A.; Thumm, U.; and Vrakking, M. J. J. What will it take to observe processes in 'real time'?, Nature Photonics 2014, 8, 162-166.

[8] Kraus, P. M.; Mignolet, B.; Baykusheva, D.; Rupenyan, A.; Horny, L.; Penka, E. F.; Grassi, G.; Tolstikhin, O. I. ; Schneider, J.; Jensen, F.; Madsen, L. B.; Bandrauk, A. D.; Remacle, F.; and Worner, H. J. Measurement and laser control of attosecond charge migration in ionized iodoacetylene, Science 2015, 350, 790-795. 
[9] Vacher, M.; Steinberg, L.; Jenkins, A. J.; Bearpark, M. J.; and Robb, M. A. Electron dynamics following photoionization: Decoherence due to the nuclear-wave-packet width, Phys. Rev. A. 2015, 92, 040502.

[10] Hentschel, M.; Kienberger, R.; Spielmann, C.; Reider, G. A.; Milosevic, N.; Brabec, T.; Corkum, P. B.; Heinzmann, U.; Drescher, M.; and Krausz, F. Attosecond metrology, Nature 2001, $414,509-513$.

[11] Paul, P. M.; Toma, E. S.; Breger, P.; Mullot, G.; Auge, F.; Balcou, P.; Muller, H. G.; and Agostini, P. Observation of a train of attosecond pulses from high harmonic generation, Science 2001, 292, 1689-1692.

[12] Drescher, M.; Hentschel, M.; Kienberger, R.; Uiberacker, M.; Yakovlev, V.; Scrinzi, A.; Westerwalbesloh, T.; Kleineberg, U.; Heinzmann, U.; and Krausz, F. Time-resolved atomic innershell spectroscopy, Nature 2002, 419, 803-807.

[13] Kling, M. F. and Vrakking, M. J. J. Attosecond electron dynamics, Annu. Rev. Phys. Chem 2008, 59, 463-492.

[14] Krausz, F. and Ivanov, M. Attosecond physics, Rev. Mod. Phys. 2009, 81, 163.

[15] Uiberacker, M.; Uphues, T.; Schultze, M.; Verhoef, A. J.; Yakovlev, V.; Kling, M. F.; Rauschenberger, J.; Kabachnik, N. M.; Schröder, H.; Lezius, M.; Kompa, K. L.; Muller, H.-G.; Vrakking, M. J. J.; Hendel, S.; Kleineberg, U.; Heinzmann, U.; Drescher, M.; and Krausz, F. Attosecond real-time observation of electron tunnelling in atoms, Nature 2007, 446, 627-632.

[16] Schultze, M.; Fiess, M.; Karpowicz, N.; Gagnon, J.; Korbman, M.; Hofstetter, M.; Neppl, S.; Cavalieri, A. L.; Komninos, Y.; Mercouris, T.; Nicolaides, C. A.; Pazourek, R.; Nagele, S.; 
Feist, J.; Burgdorfer, J.; Azzeer, A. M.; Ernstorfer, R.; Kienberger, R.; Kleineberg, U.;

Goulielmakis, E.; Krausz, F.; and Yakovlev, V. S. Delay in photoemission, Science, 2010, 328, $1658-1662$.

[17] Sansone, G.; Kelkensberg, F.; Pérez-Torres, J. F.; Morales, F.; Kling, M. F.; Siu, W.; Ghafur, O.; Johnsson, P.; Swoboda, M.; Benedetti, E.; Ferrari, F.; Lépine, F.; Sanz-Vicario, J. L.; Zherebtsov, S. Znakovskaya, I.; L'Huillier, A.; Ivanov, M. Y.; Nisoli, M.; Martín, F.; and Vrakking, M. J. J. Electron localization following attosecond molecular photoionization, Nature 2010, 465, 763-766.

[18] Ranitovic, P.; Hogle, C. W. ; Riviére, P.; Palacios, A.; Tong, X.-M.; Toshima, N.; GonzálezCastrillo, A.; Martin, L.; Martín, F.; Murnane, M. M.; and Kapteyn, H. Attosecond vacuum UV coherent control of molecular dynamics, Proc. Natl. Acad. Sci. USA 2014, 111, 912-917.

[19] Belshaw, L.; Calegari, F.; Duffy, M. J.; Trabattoni, A.; Poletto, L.; Nisoli, M.; and Greenwood, J. B. Observation of ultrafast charge migration in an amino acid, J. Phys. Chem. Lett. 2012, 3, 3751-3754.

[20] Goulielmakis, E.; Loh, Z-H.; Wirth, A.; Santra, R.; Rohringer, N.; Yakovlev, V. S.; Zherebtsov, S.; Pfeifer, T.; Azzeer, A. M.; Kling, M. F.; Leone, S. R.; and Krausz, F. Real-time observation of valence electron motion, Nature 2010, 466, 739-743.

[21] Calegari, F.; Ayuso, D.; Trabattoni, A.; Belshaw, L.; De Camillis, S.; Frassetto, F.; Poletto, L.; Palacios, A.; Decleva, P.; Greenwood, J. B.; Martín, F.; and Nisoli, M. Ultrafast charge dynamics in an amino acid induced by attosecond pulses, IEEE J. Sel. Top. Quantum Electron. 2015, 21, 8700512 . 
[22] Arnold, C.; Vendrell, O.; and Santra, R. Electronic decoherence following photoionization: full quantum-dynamical treatment of the influence of nuclear motion, Phys. Rev. A 2017, 95, 033425.

[23] Vacher, M.; Bearpark, M. J.; Robb, M. A.; and Malhado, J. P. Electron dynamics upon ionization of polyatomic molecules: coupling to quantum nuclear motion and decoherence, Phys. Rev. Lett. 2017, 118, 083001.

[24] Galbraith, M. C. E.; Scheit, S.; Golubev, N. V .; Reitsma, G.; Zhavoronkov, N.; Despré, V.; Lépine, F.; Kuleff, A. I.; Vrakking, M. J. J.; Kornilov, O.; Köppel, H.; and Mikosch, J. Fewfemtosecond passage of conical intersections in the benzene cation, Nature Comm. 2017, 8, 1018.

[25] Gonnelli, M. and Strambini, G. B. Phosphorescence lifetime of tryptophan in proteins, Biochemistry 1995, 34, 13847-13857.

[26] Vanderkooi, J. M. in: Lakovitch, J. R. (ed.), Topics in fluorescence spectroscopy, Vol. 3, Plenum Press, New York-London 1992, 113-136.

[27] Shih, C.; Museth, A. K.; Abrahamsson, M.; Blanco-Rodriguez, A. M.; Di Bilio, A. J.; Sudhamsu, J.; Crane, B. R.; Ronayne, K. L.; Towrie, M.; Vlcek Jr, A.; Richards, J. H.; Winkler, J. R.; and Gray, H. B. Tryptophan-accelerated electron flow through proteins, Science 2008, 320, 1760-1762.

[28] Toffoli, G. F. D.; Stener, M.; and Decleva, P. Convergence of the multicenter B-spline DFT approach for the continuum, Chem. Phys. 2002, 276, 25-43. 
[29] Stener, M.; Furlan, S.; and Decleva, P. Density functional calculations of photoionization with an exchange-correlation potential with the correct asymptotic behaviour, J. Phys. B: At. Mol. Opt. Phys. 2000, 33, 1081.

[30] M. Stener, D. Toffoli, G. Fronzoni, and P. Decleva, Recent advances in molecular photoionization by density functional theory-based approaches, Theoret. Chem. Acc. 117, 943956 (2007).

[31] F. Calegari, D. Ayuso, A. Trabattoni, L. Belshaw, S. De Camillis, F. Frassetto, L. Poletto, A. Palacios, P. Decleva, J. Greenwood, F. Martín, and M. Nisoli, Ultrafast charge dynamics in an amino acid induced by attosecond pulses, IEEE Journal of Selected Topics in Quantum Electronics 21, $8700512(2015)$.

[32] Ayuso, D.; Palacios, A.; Decleva, P.; and Martín, F. Ultrafast charge dynamics in glycine induced by attosecond pulses, Ultrafast charge dynamics in glycine induced by attosecond pulses, Phys. Chem. Chem. Phys. 2017, 19, 19767-19776.

[33] Plekan, O.; Feyer, V.; Richter, R.; Coreno, M.; and Prince, K. C. Valence photoionization and photofragmentation of aromatic amino acids, Mol. Phys. 2008, 106, 1143-1153.

[34] CPMD, copyright IBM corp 1990-2015, copyright MPI für festkörperforschung

Stuttgart 1997-2001, 2014. http://www.cpmd.org/.

[35] Ott, C.; Kaldun, A.; Argenti, L.; Raith, P.; Meyer, K.; Laux, M.; Zhang, Y.; Bláttermann, A.; Hagstotz, S.; Ding, T.; Heck, R.; Madroñero, J.; Martín, F.; and Pfeifer, T. Reconstruction and control of a time-dependent two-electron wave packet, Nature 2014, 516, 374-378. 
[36] Lara-Astiaso, M.; Palacios, A.; Decleva, P.; Tavernelli, I.; and Martín, F. Role of electronnuclear coupled dynamics on charge migration induced by attosecond pulses in glycine, Chem. Phys. Lett. 2017, 683, 357-364.

[37] Lara-Astiaso, M.; Ayuso, D.; Tavernelli, I.; Decleva, P.; Palacios, A.; and Martín, F. Decoherence, control and attosecond probing of XUV-induced charge migration in biomolecules. A theoretical outlook, Faraday Discuss. 2016, 194, 41-59.

[38] Troullier, N. and Martins, J. L. Efficient pseudopotentials for plane-wave calculations, Phys. Rev. B 1991, 43, 1993-2006.

[39] Kleinman, L. and Bylander, D. M. Efficacious form for model pseudopotentials, Phys. Rev. Lett. 1982, 48, 1425-1428.

[40] Drobowolski, J. C.; Lipinski, P. F. J; Rude, J. E.; and Sadlej, J. in Optical Spectroscopy and Computational Methods in Biology and Medicine, Challenges and Advances in Computational Chemistry and Physics 14, M. Baranska Ed., Springer, London 2014, 83-160.

[41] Curchod, B. F. E.; Rothlisberger, U.; and Tavernelli, I. Trajectory-based nonadiabatic dynamics with time-dependent density functional theory, ChemPhysChem 2013, 14, 1314-1340. 